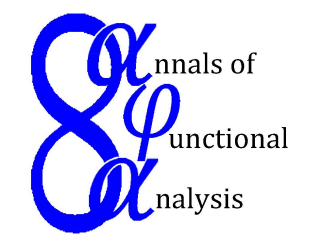

Ann. Funct. Anal. 6 (2015), no. 2, 224-233

http://doi.org/10.15352/afa/06-2-19

ISSN: 2008-8752 (electronic)

http://projecteuclid.org/afa

\title{
SOME CHARACTERIZATIONS OF HERZ-TYPE HARDY SPACES WITH VARIABLE EXPONENT
}

\author{
HONGBIN WANG ${ }^{*}$ AND ZONGGUANG LIU² \\ Communicated by J. Soria
}

\begin{abstract}
In this paper, the authors establish some real-variable characterizations of Herz-type Hardy spaces with variable exponent.
\end{abstract}

\section{INTRODUCTION AND PRELIMINARIES}

Given an open set $\Omega \subset \mathbb{R}^{n}$, and a measurable function $p(\cdot): \Omega \rightarrow[1, \infty)$, $L^{p(\cdot)}(\Omega)$ denotes the set of measurable functions $f$ on $\Omega$ such that for some $\lambda>0$,

$$
\int_{\Omega}\left(\frac{|f(x)|}{\lambda}\right)^{p(x)} d x<\infty .
$$

This set becomes a Banach function space when equipped with the LuxemburgNakano norm

$$
\|f\|_{L^{p(\cdot)}(\Omega)}=\inf \left\{\lambda>0: \int_{\Omega}\left(\frac{|f(x)|}{\lambda}\right)^{p(x)} d x \leq 1\right\} .
$$

These spaces are referred to as variable Lebesgue spaces or, more simply, as variable $L^{p}$ spaces, since they generalized the standard $L^{p}$ spaces: if $p(x)=p$ is constant, then $L^{p(\cdot)}(\Omega)$ is isometrically isomorphic to $L^{p}(\Omega)$. The $L^{p}$ spaces with variable exponent are a special case of Musielak-Orlicz spaces.

For all compact subsets $E \subset \Omega$, the space $L_{\text {loc }}^{p(\cdot)}(\Omega)$ is defined by $L_{\text {loc }}^{p(\cdot)}(\Omega):=$ $\left\{f: f \in L^{p(\cdot)}(E)\right\}$. Define $\mathcal{P}(\Omega)$ to be the set of $p(\cdot): \Omega \rightarrow[1, \infty)$ such that

$$
p^{-}=\operatorname{essinf}\{p(x): x \in \Omega\}>1, \quad p^{+}=\operatorname{ess} \sup \{p(x): x \in \Omega\}<\infty .
$$

Date: Received: Jun. 2, 2014; Accepted: Sep. 16, 2014.

* Corresponding author.

2010 Mathematics Subject Classification. Primary 46E30; Secondary 42B30, 42B35.

Key words and phrases. Herz-type Hardy space, variable exponent, real-variable characterization. 
Denote $p^{\prime}(x)=p(x) /(p(x)-1)$. Let $\mathcal{B}(\Omega)$ be the set of $p(\cdot) \in \mathcal{P}(\Omega)$ such that the Hardy-Littlewood maximal operator $M$ is bounded on $L^{p(\cdot)}(\Omega)$.

In variable $L^{p}$ spaces there are some important lemmas as follows.

Lemma 1.1. ([4]) Let $p(\cdot) \in \mathcal{P}(\Omega)$. If $f \in L^{p(\cdot)}(\Omega)$ and $g \in L^{p^{\prime}(\cdot)}(\Omega)$, then $f g$ is integrable on $\Omega$ and

$$
\int_{\Omega}|f(x) g(x)| d x \leq r_{p}\|f\|_{L^{p(\cdot)}(\Omega)}\|g\|_{L^{p^{(}(\cdot)}(\Omega)}
$$

where

$$
r_{p}=1+1 / p^{-}-1 / p^{+}
$$

This inequality is named the generalized Hölder inequality with respect to the variable $L^{p}$ spaces.

Lemma 1.2. ([1]) Given a set $\Omega$ with finite measure, and exponent functions $p(\cdot), q(\cdot): \Omega \rightarrow[1, \infty)$ such that $p(x) \leq q(x)$,

$$
\|f\|_{L^{p(\cdot)}(\Omega)} \leq C(1+|\Omega|)\|f\|_{L^{q(\cdot)}(\Omega)}
$$

Lemma 1.3. ([3]) Let $p(\cdot) \in \mathcal{B}\left(\mathbb{R}^{n}\right)$. Then there exists a positive constant $C$ such that for all balls $B$ in $\mathbb{R}^{n}$ and all measurable subsets $S \subset B$,

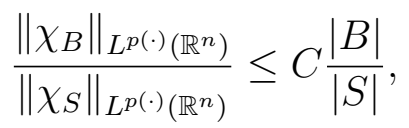

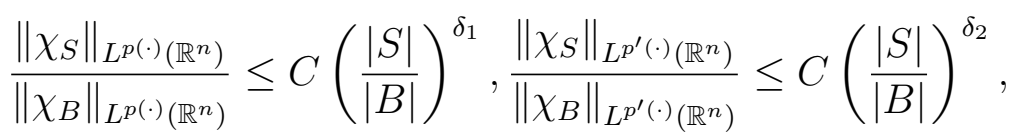

where $0<\delta_{1}, \delta_{2}<1$ are constants.

Throughout this paper $\delta_{1}$ and $\delta_{2}$ are the same as in Lemma 1.3.

Lemma 1.4. ([3]) Suppose $p(\cdot) \in \mathcal{B}\left(\mathbb{R}^{n}\right)$. Then there exists a constant $C>0$ such that for all balls $B$ in $\mathbb{R}^{n}$,

$$
\frac{1}{|B|}\left\|\chi_{B}\right\|_{L^{p(\cdot)\left(\mathbb{R}^{n}\right)}}\left\|\chi_{B}\right\|_{L^{p^{\prime}(\cdot)\left(\mathbb{R}^{n}\right)}} \leq C .
$$

Firstly we give the definition of the Herz spaces with variable exponent. Let $B_{k}=\left\{x \in \mathbb{R}^{n}:|x| \leq 2^{k}\right\}$ and $A_{k}=B_{k} \backslash B_{k-1}$ for $k \in \mathbb{Z}$. Denote $\mathbb{Z}_{+}$and $\mathbb{N}$ as the sets of all positive and non-negative integers, $\chi_{k}=\chi_{A_{k}}$ for $k \in \mathbb{Z}, \tilde{\chi}_{k}=\chi_{k}$ if $k \in \mathbb{Z}_{+}$and $\tilde{\chi}_{0}=\chi_{B_{0}}$, where $\chi_{A_{k}}$ is the characteristic function of $A_{k}$.

Definition 1.5. ([3]) Let $\alpha \in \mathbb{R}, 0<p \leq \infty$ and $q(\cdot) \in \mathcal{P}\left(\mathbb{R}^{n}\right)$. The homogeneous Herz space $\dot{K}_{q(\cdot)}^{\alpha, p}\left(\mathbb{R}^{n}\right)$ is defined by

$$
\dot{K}_{q(\cdot)}^{\alpha, p}\left(\mathbb{R}^{n}\right)=\left\{f \in L_{\mathrm{loc}}^{q(\cdot)}\left(\mathbb{R}^{n} \backslash\{0\}\right):\|f\|_{\dot{K}_{q(\cdot)}^{\alpha, p}\left(\mathbb{R}^{n}\right)}<\infty\right\},
$$

where

$$
\|f\|_{\dot{K}_{q(\cdot)}^{\alpha, p}\left(\mathbb{R}^{n}\right)}=\left\{\sum_{k \in \mathbb{Z}} 2^{k \alpha p}\left\|f \chi_{k}\right\|_{L^{q(\cdot)}\left(\mathbb{R}^{n}\right)}^{p}\right\}^{1 / p} .
$$


The non-homogeneous Herz space $K_{q(\cdot)}^{\alpha, p}\left(\mathbb{R}^{n}\right)$ is defined by

$$
K_{q(\cdot)}^{\alpha, p}\left(\mathbb{R}^{n}\right)=\left\{f \in L_{\mathrm{loc}}^{q(\cdot)}\left(\mathbb{R}^{n}\right):\|f\|_{K_{q(\cdot)}^{\alpha, p}\left(\mathbb{R}^{n}\right)}<\infty\right\}
$$

where

$$
\|f\|_{K_{q(\cdot)}^{\alpha, p}\left(\mathbb{R}^{n}\right)}=\left\{\sum_{k=0}^{\infty} 2^{k \alpha p}\left\|f \tilde{\chi}_{k}\right\|_{L^{q(\cdot)}\left(\mathbb{R}^{n}\right)}^{p}\right\}^{1 / p} .
$$

In [6], we establish the following boundedness theorem on the Herz spaces with variable exponent for a class of sublinear operators.

Lemma 1.6. ([6]) Let $0<\alpha<n \delta_{2}, 0<p<\infty$ and $q(\cdot) \in \mathcal{B}\left(\mathbb{R}^{n}\right)$. If a sublinear operator $T$ satisfies

$$
|T f(x)| \leq C\|f\|_{1} /|x|^{n}, \quad \text { if } \quad \operatorname{dist}(x, \operatorname{supp} f)>|x| / 2,
$$

for any integrable function $f$ with a compact support and $T$ is bounded on $L^{q(\cdot)}\left(\mathbb{R}^{n}\right)$, then $T$ is bounded on $\dot{K}_{q(\cdot)}^{\alpha, p}\left(\mathbb{R}^{n}\right)$ and $K_{q(\cdot)}^{\alpha, p}\left(\mathbb{R}^{n}\right)$, respectively.

In [7], we gave the definition of Herz-type Hardy space with variable exponent $H \dot{K}_{q(\cdot)}^{\alpha, p}\left(\mathbb{R}^{n}\right) . \mathcal{S}\left(\mathbb{R}^{n}\right)$ denotes the space of Schwartz functions, and $\mathcal{S}^{\prime}\left(\mathbb{R}^{n}\right)$ denotes the dual space of $\mathcal{S}\left(\mathbb{R}^{n}\right)$. Let $G_{N} f(x)$ be the grand maximal function of $f(x)$ defined by

$$
G_{N} f(x)=\sup _{\phi \in \mathcal{A}_{N}}\left|\phi_{\nabla}^{*}(f)(x)\right|,
$$

where $\mathcal{A}_{N}=\left\{\phi \in \mathcal{S}\left(\mathbb{R}^{n}\right): \sup _{|\alpha|,|\beta| \leq N}\left|x^{\alpha} D^{\beta} \phi(x)\right| \leq 1\right\}$ and $N>n+1, \phi_{\nabla}^{*}$ is the nontangential maximal operator defined by

$$
\phi_{\nabla}^{*}(f)(x)=\sup _{|y-x|<t}\left|\phi_{t} * f(y)\right|
$$

with $\phi_{t}(x)=t^{-n} \phi(x / t)$.

Definition 1.7. ([7]) Let $\alpha \in \mathbb{R}, 0<p<\infty, q(\cdot) \in \mathcal{P}\left(\mathbb{R}^{n}\right)$ and $N>n+1$.

(i)The homogeneous Herz-type Hardy space $H \dot{K}_{q(\cdot)}^{\alpha, p}\left(\mathbb{R}^{n}\right)$ is defined by

$$
H \dot{K}_{q(\cdot)}^{\alpha, p}\left(\mathbb{R}^{n}\right)=\left\{f \in \mathcal{S}^{\prime}\left(\mathbb{R}^{n}\right): G_{N} f(x) \in \dot{K}_{q(\cdot)}^{\alpha, p}\left(\mathbb{R}^{n}\right)\right\}
$$

and we define $\|f\|_{H \dot{K}_{q(\cdot)}^{\alpha, p}\left(\mathbb{R}^{n}\right)}=\left\|G_{N} f\right\|_{\dot{K}_{q(\cdot)}^{\alpha, p}\left(\mathbb{R}^{n}\right)}$.

(ii)The non-homogeneous Herz-type Hardy space $H K_{q(\cdot)}^{\alpha, p}\left(\mathbb{R}^{n}\right)$ is defined by

$$
H K_{q(\cdot)}^{\alpha, p}\left(\mathbb{R}^{n}\right)=\left\{f \in \mathcal{S}^{\prime}\left(\mathbb{R}^{n}\right): G_{N} f(x) \in K_{q(\cdot)}^{\alpha, p}\left(\mathbb{R}^{n}\right)\right\}
$$

and we define $\|f\|_{H K_{q(\cdot)}^{\alpha, p}\left(\mathbb{R}^{n}\right)}=\left\|G_{N} f\right\|_{K_{q(\cdot)}^{\alpha, p}\left(\mathbb{R}^{n}\right)}$.

Let us explain the outline of this paper. In Section 2 we will prove some properties for $\dot{K}_{q(\cdot)}^{\alpha, p}\left(\mathbb{R}^{n}\right)$ and $K_{q(\cdot)}^{\alpha, p}\left(\mathbb{R}^{n}\right)$. We will give our main result in Section 3, that is some real-variable characterizations of the Herz-type Hardy space with variable exponent. 


\section{Some properties for Herz SPACES With VARIABle EXPONENT}

We first give the following properties for $\dot{K}_{q(\cdot)}^{\alpha, p}\left(\mathbb{R}^{n}\right)$ and $K_{q(\cdot)}^{\alpha, p}\left(\mathbb{R}^{n}\right)$.

Theorem 2.1. Let $\alpha \in \mathbb{R}, 0<p \leq \infty$ and $q(\cdot) \in \mathcal{P}\left(\mathbb{R}^{n}\right)$. Then we have

(1) if $p_{1} \leq p_{2}$, then $\dot{K}_{q(\cdot)}^{\alpha, p_{1}}\left(\mathbb{R}^{n}\right) \subset \dot{K}_{q(\cdot)}^{\alpha, p_{2}}\left(\mathbb{R}^{n}\right)$ and $K_{q(\cdot)}^{\alpha, p_{1}}\left(\mathbb{R}^{n}\right) \subset K_{q(\cdot)}^{\alpha, p_{2}}\left(\mathbb{R}^{n}\right)$.

(2) if $0<\alpha_{2} \leq \alpha_{1}$, then $K_{q(\cdot)}^{\alpha_{1}, p}\left(\mathbb{R}^{n}\right) \subset K_{q(\cdot)}^{\alpha_{2}, p}\left(\mathbb{R}^{n}\right)$.

(3) if $\Omega \subset \mathbb{R}^{n},|\Omega|<\infty$ and $q_{1}(\cdot), q_{2}(\cdot) \in \mathcal{P}(\Omega)$ such that $q_{2}(\cdot) \leq q_{1}(\cdot)$, then

$$
\dot{K}_{q_{1}(\cdot)}^{\alpha, p}(\Omega) \subset \dot{K}_{q_{2}(\cdot)}^{\alpha, p}(\Omega), K_{q_{1}(\cdot)}^{\alpha, p}(\Omega) \subset K_{q_{2}(\cdot)}^{\alpha, p}(\Omega) .
$$

Proof. We first consider (1). It suffices to prove the property for the homogeneous case. The non-homogeneous case can be proved in the same way. Note that $p_{1} \leq p_{2}$ and

$$
\left(\sum_{k=1}^{\infty}\left|a_{k}\right|\right)^{r} \leq \sum_{k=1}^{\infty}\left|a_{k}\right|^{r}, \quad 0<r \leq 1
$$

So we have

$$
\begin{aligned}
\|f\|_{\dot{K}_{q(\cdot)}^{\alpha, p_{2}}\left(\mathbb{R}^{n}\right)} & =\left\{\sum_{k=-\infty}^{\infty} 2^{k \alpha p_{1} \cdot \frac{p_{2}}{p_{1}}}\left\|f \chi_{k}\right\|_{L^{q(\cdot)}\left(\mathbb{R}^{n}\right)}^{p_{1} \cdot \frac{p_{2}}{p_{1}}}\right\}^{\frac{1}{p_{1} \cdot \frac{p_{1}}{p_{2}}}} \\
& \leq\left\{\sum_{k=-\infty}^{\infty} 2^{k \alpha p_{1}}\left\|f \chi_{k}\right\|_{L^{q(\cdot)}\left(\mathbb{R}^{n}\right)}^{p_{1}}\right\}^{\frac{1}{p_{1}}} \\
& =\|f\|_{\dot{K}_{q(\cdot)}^{\alpha, p_{1}}\left(\mathbb{R}^{n}\right)}^{\infty}
\end{aligned}
$$

That is $\dot{K}_{q(\cdot)}^{\alpha, p_{1}}\left(\mathbb{R}^{n}\right) \subset \dot{K}_{q(\cdot)}^{\alpha, p_{2}}\left(\mathbb{R}^{n}\right)$.

Now we see (2). Note that $0<\alpha_{2} \leq \alpha_{1}$, so by the Hölder inequality we have

$$
\begin{aligned}
& \|f\|_{K_{q(\cdot)}^{\alpha_{2}, p}\left(\mathbb{R}^{n}\right)}=\left\{\sum_{k=0}^{\infty} 2^{k \alpha_{2} p}\left\|f \tilde{\chi}_{k}\right\|_{L^{q(\cdot)}\left(\mathbb{R}^{n}\right)}^{p\left(\frac{\alpha_{2}}{\alpha_{1}}+\frac{\alpha_{1}-\alpha_{2}}{\alpha_{1}}\right)}\right\}^{1 / p} \\
& \leq\left\{C\left(\sum_{k=0}^{\infty}\left(2^{k \alpha_{2} p}\left\|f \tilde{\chi}_{k}\right\|_{L^{q(\cdot)}\left(\mathbb{R}^{n}\right)}^{p \frac{\alpha_{2}}{\alpha_{1}}}\right)^{\frac{\alpha_{1}}{\alpha_{2}}}\right)^{\frac{\alpha_{2}}{\alpha_{1}}}\right. \\
& \left.\times\left(\sum_{k=0}^{\infty}\left\|f \tilde{\chi}_{k}\right\|_{L^{q(\cdot)\left(\mathbb{R}^{n}\right)}}^{\frac{\alpha_{1}-\alpha_{2}}{\alpha_{2}}\left(\frac{\alpha_{1}}{\alpha_{2}}\right)^{\prime}}\right)^{\frac{1}{\left(\frac{\alpha_{1}}{\alpha_{2}}\right)^{\prime}}}\right\}^{1 / p} \\
& \begin{array}{l}
\leq\left\{C\left(\sum_{k=0}^{\infty} 2^{k \alpha_{1} p}\left\|f \tilde{\chi}_{k}\right\|_{L^{q(\cdot)}\left(\mathbb{R}^{n}\right)}^{p}\right)^{\frac{\alpha_{2}}{\alpha_{1}}}\left(\sum_{k=0}^{\infty}\left\|f \tilde{\chi}_{k}\right\|_{L^{q(\cdot)}\left(\mathbb{R}^{n}\right)}^{p}\right)^{\frac{\alpha_{1}-\alpha_{2}}{\alpha_{1}}}\right\}^{1 / p} \\
\leq C\|f\|_{K_{q(\cdot)}^{\alpha_{1}, p}\left(\mathbb{R}^{n}\right) .}
\end{array}
\end{aligned}
$$

That is $K_{q(\cdot)}^{\alpha_{1}, p}\left(\mathbb{R}^{n}\right) \subset K_{q(\cdot)}^{\alpha_{2}, p}\left(\mathbb{R}^{n}\right)$. 
Next we estimate (3). It suffices to prove the property for the homogeneous case. By Lemma 1.2 we have

$$
\begin{aligned}
\|f\|_{\dot{K}_{q_{2}(\cdot)}^{\alpha, p}(\Omega)} & =\left\{\sum_{k=-\infty}^{\infty} 2^{k \alpha p}\left\|f \chi_{k}\right\|_{L^{q_{2}(\cdot)}(\Omega)}^{p}\right\}^{1 / p} \\
& \leq C(1+|\Omega|)\left\{\sum_{k=-\infty}^{\infty} 2^{k \alpha p}\left\|f \chi_{k}\right\|_{L^{q_{1}(\cdot)}(\Omega)}^{p}\right\}^{1 / p} \\
& \leq C\|f\|_{\dot{K}_{q_{1}(\cdot)}^{\alpha, p}(\Omega)} .
\end{aligned}
$$

That is $\dot{K}_{q_{1}(\cdot)}^{\alpha, p}(\Omega) \subset \dot{K}_{q_{2}(\cdot)}^{\alpha, p}(\Omega)$.

Thus we complete the proof of Theorem 2.1.

Theorem 2.2. Let $0<\alpha<\infty, 0<p \leq \infty$ and $q(\cdot) \in \mathcal{P}\left(\mathbb{R}^{n}\right)$. Then

$$
K_{q(\cdot)}^{\alpha, p}\left(\mathbb{R}^{n}\right) \supset \dot{K}_{q(\cdot)}^{\alpha, p}\left(\mathbb{R}^{n}\right) \cap L^{q(\cdot)}\left(\mathbb{R}^{n}\right)
$$

and for $f \in \dot{K}_{q(\cdot)}^{\alpha, p}\left(\mathbb{R}^{n}\right) \cap L^{q(\cdot)}\left(\mathbb{R}^{n}\right)$,

$$
\|f\|_{K_{q(\cdot)}^{\alpha, p}\left(\mathbb{R}^{n}\right)} \leq\|f\|_{\dot{K}_{q(\cdot)}^{\alpha, p}\left(\mathbb{R}^{n}\right)}+\|f\|_{L^{q(\cdot)}\left(\mathbb{R}^{n}\right)} .
$$

Proof. If $f \in \dot{K}_{q(\cdot)}^{\alpha, p}\left(\mathbb{R}^{n}\right) \cap L^{q(\cdot)}\left(\mathbb{R}^{n}\right)$, then

$$
\begin{aligned}
\|f\|_{K_{q(\cdot)}^{\alpha, p}\left(\mathbb{R}^{n}\right)} & =\|f\|_{L^{q(\cdot)}(|x| \leq 1)}^{p}+\sum_{k=1}^{\infty} 2^{k \alpha p}\left\|f \chi_{k}\right\|_{L^{q(\cdot)}\left(\mathbb{R}^{n}\right)}^{p} \\
& \leq\|f\|_{L^{q(\cdot)}\left(\mathbb{R}^{n}\right)}^{p}+\|f\|_{\dot{K}_{q(\cdot)}^{\alpha, p}\left(\mathbb{R}^{n}\right)^{\prime}}^{p}
\end{aligned}
$$

This finishes the proof of Theorem 2.2.

\section{Some ReAl-VARiable characterizations for Herz-type Hardy SPACES WITH VARIABLE EXPONENT}

By Theorem 2.2 and the $L^{q(\cdot)}\left(\mathbb{R}^{n}\right)$-boundedness $\left(q(\cdot) \in \mathcal{B}\left(\mathbb{R}^{n}\right)\right)$ of the grand maximal operator $G_{N}$, it is easy to deduce the following conclusion.

Theorem 3.1. Let $0<\alpha<\infty, 0<p<\infty$ and $q(\cdot) \in \mathcal{B}\left(\mathbb{R}^{n}\right)$. Then

$$
H K_{q(\cdot)}^{\alpha, p}\left(\mathbb{R}^{n}\right) \supset H \dot{K}_{q(\cdot)}^{\alpha, p}\left(\mathbb{R}^{n}\right) \cap L^{q(\cdot)}\left(\mathbb{R}^{n}\right)
$$

To give some real-variable characterizations for $H \dot{K}_{q(\cdot)}^{\alpha, p}\left(\mathbb{R}^{n}\right)$ and $H K_{q(\cdot)}^{\alpha, p}\left(\mathbb{R}^{n}\right)$, we first introduce some maximal operator.

Let $\phi \in \mathcal{S}\left(\mathbb{R}^{n}\right)$ with integral 1. For $t>0$, set $\phi_{t}(x)=t^{-n} \phi(x / t)$. For $f \in$ $\mathcal{S}^{\prime}\left(\mathbb{R}^{n}\right)$, define the maximal operator $\phi_{+}^{*}$ by

$$
\phi_{+}^{*}(f)(x)=\sup _{t>0}\left|\left(f * \phi_{t}\right)(x)\right| .
$$

Also, we define the maximal operator $\phi_{\nabla, N}^{*}($ with $N>1)$ and $\phi_{M}^{* *}\left(\right.$ with $M \in \mathbb{Z}_{+}$) by

$$
\phi_{\nabla, N}^{*}(f)(x)=\sup _{t>0} \sup _{|x-y|<N t}\left|\left(f * \phi_{t}\right)(y)\right|
$$


and

$$
\phi_{M}^{* *}(f)(x)=\sup _{(y, t) \in \mathbb{R}_{+}^{n+1}}\left|\left(f * \phi_{t}\right)(y)\right|\left(\frac{t}{|x-y|+t}\right)^{M} .
$$

About the relation of these operators, we first have

Lemma 3.2. ([2]) If $N \geq M+n+1$, then there exists a constant $C$ such that

$$
G_{N}(f)(x) \leq C \phi_{M}^{* *}(f)(x) .
$$

Next we give the following characterization theorem.

Theorem 3.3. Let $0<\alpha<\infty, 0<p<\infty$ and $q(\cdot) \in \mathcal{B}\left(\mathbb{R}^{n}\right)$. For $f \in \mathcal{S}^{\prime}\left(\mathbb{R}^{n}\right)$, the following statements are equivalent:

(i) $f \in H \dot{K}_{q(\cdot)}^{\alpha, p}\left(\mathbb{R}^{n}\right) \quad\left(\right.$ or $\left.H K_{q(\cdot)}^{\alpha, p}\left(\mathbb{R}^{n}\right)\right)$.

(ii) For some $N>1, \phi_{\nabla, N}^{*}(f) \in \dot{K}_{q(\cdot)}^{\alpha, p}\left(\mathbb{R}^{n}\right) \quad\left(\right.$ or $\left.K_{q(\cdot)}^{\alpha, p}\left(\mathbb{R}^{n}\right)\right)$.

(iii) $\phi_{\nabla}^{*}(f) \in \dot{K}_{q(\cdot)}^{\alpha, p}\left(\mathbb{R}^{n}\right) \quad$ or $\left.K_{q(\cdot)}^{\alpha, p}\left(\mathbb{R}^{n}\right)\right)$.

(iv) $\phi_{+}^{*}(f) \in \dot{K}_{q(\cdot)}^{\alpha, p}\left(\mathbb{R}^{n}\right) \quad\left(\right.$ or $\left.K_{q(\cdot)}^{\alpha, p}\left(\mathbb{R}^{n}\right)\right)$.

Proof. We only prove the homogeneous case. The non-homogeneous case is similar. Note that

$$
\phi_{\nabla, N}^{*}(f)(x) \geq \phi_{\nabla}^{*}(f)(x) \geq \phi_{+}^{*}(f)(x)
$$

and that for any $N>n+1$,

$$
\phi_{\nabla}^{*}(f)(x) \leq C G_{N}(f)(x)
$$

It is obvious that (ii) $\Rightarrow($ iii $) \Rightarrow($ iv) and (i) $\Rightarrow$ (iii). Thus, it suffices to prove that (iv) $\Rightarrow$ (ii) and (iv) $\Rightarrow$ (i).

We first prove (iv) $\Rightarrow\left(\right.$ ii). For $l, N \in \mathbb{Z}_{+}$, define

$$
u_{\varepsilon, l, N}^{*}(x)=\sup _{|x-y|<N t<1 / \varepsilon}\left|\left(f * \phi_{t}\right)(y)\right|\left(\frac{N t}{N t+\varepsilon}\right)^{l}(1+\varepsilon N|y|)^{-l} .
$$

By the Fatou lemma of series and integration, we need only to show that for any $r \in(0,1)$

$$
\left\|u_{\varepsilon, l, N}^{*}\right\|_{\dot{K}_{q(\cdot)}^{\alpha, p}\left(\mathbb{R}^{n}\right)} \leq C N^{n / r}\left\|\phi_{+}^{*}(f)\right\|_{\dot{K}_{q(\cdot)}^{\alpha, p}\left(\mathbb{R}^{n}\right)} .
$$

Let

$$
U_{\varepsilon, l, N}^{*}(x)=\sup _{|x-y|<N t<1 / \varepsilon} t\left|\nabla_{y}\left(f * \phi_{t}\right)(y)\right|\left(\frac{N t}{N t+\varepsilon}\right)^{l}(1+\varepsilon N|y|)^{-l} .
$$

As in [5], if $l$ is large enough, then for any $p_{1} \in(0,1)$ we have

$$
U_{\varepsilon, l, N}^{*}(x) \leq C\left(M\left[\left(u_{\varepsilon, l, N}^{*}\right)^{p_{1}}\right](x)\right)^{1 / p_{1}},
$$

where $M$ is the Hardy-Littlewood maximal operator, and $C$ is independent of $\varepsilon, N$ and $f$. Set $E_{\varepsilon}=\left\{x: U_{\varepsilon, l, N}^{*}(x) \leq C_{0} u_{\varepsilon, l, N}^{*}(x)\right\}$ and $E_{\varepsilon}^{c}=\mathbb{R}^{n} \backslash E_{\varepsilon}$, where 
$C_{0}$ is a positive constant which will be chosen later. Take $p_{1} \in(0,1)$ such that $0<p_{1} \alpha<n \delta_{2}$, then by Lemma 1.6 we have

$$
\begin{aligned}
\left\|u_{\varepsilon, l, N}^{*} \chi_{E_{\varepsilon}^{c}}\right\|_{\dot{K}_{q(\cdot)}^{\alpha, p}\left(\mathbb{R}^{n}\right)} & \leq C_{0}^{-1}\left\|U_{\varepsilon, l, N}^{*}\right\|_{\dot{K}_{q(\cdot)}^{\alpha, p}\left(\mathbb{R}^{n}\right)} \\
& \leq C C_{0}^{-1}\left\|M\left[\left(u_{\varepsilon, l, N}^{*}\right)^{p_{1}}\right]\right\|_{\dot{K}_{q(\cdot) / p_{1}}^{\alpha / p_{1}}\left(\mathbb{R}^{\prime}, p_{1}\right.}^{1}\left(\mathbb{R}^{n}\right) \\
& \leq C C_{0}^{-1}\left\|\left(u_{\varepsilon, l, N}^{*}\right)^{p_{1}}\right\|_{\dot{K}_{q(\cdot) / p_{1}}^{\alpha / p_{1}, p / p_{1}}\left(\mathbb{R}^{n}\right)}^{1 / p_{1}} \\
& =C C_{0}^{-1}\left\|u_{\varepsilon, l, N}^{*}\right\|_{\dot{K}_{q(\cdot)}^{\alpha, p}\left(\mathbb{R}^{n}\right)} \cdot
\end{aligned}
$$

Therefore,

$$
\begin{aligned}
\left\|u_{\varepsilon, l, N}^{*}\right\|_{\dot{K}_{q(\cdot)}^{\alpha, p}\left(\mathbb{R}^{n}\right)} & \leq\left\|u_{\varepsilon, l, N}^{*} \chi_{E_{\varepsilon}}\right\|_{\dot{K}_{q(\cdot)}^{\alpha, p}\left(\mathbb{R}^{n}\right)}+\left\|u_{\varepsilon, l, N}^{*} \chi_{E_{\varepsilon}^{c}}\right\|_{\dot{K}_{q, \cdot)}^{\alpha, p}\left(\mathbb{R}^{n}\right)} \\
& \leq\left\|u_{\varepsilon, l, N}^{*} \chi_{E_{\varepsilon}}\right\|_{\dot{K}_{q(\cdot)}^{\alpha, p}\left(\mathbb{R}^{n}\right)}+C C_{0}^{-1}\left\|u_{\varepsilon, l, N}^{*}\right\|_{\dot{K}_{q(\cdot)}^{\alpha, p}\left(\mathbb{R}^{n}\right)} \\
& \leq 2\left\|u_{\varepsilon, l, N}^{*} \chi_{E_{\varepsilon}}\right\|_{\dot{K}_{q(\cdot)}^{\alpha, p}\left(\mathbb{R}^{n}\right)}
\end{aligned}
$$

if we choose $C_{0}$ large enough. Thus, the proof that (iv) $\Rightarrow$ (ii) can be reduced to prove that

$$
\left\|u_{\varepsilon, l, N}^{*} \chi_{E_{\varepsilon}}\right\|_{\dot{K}_{q(\cdot)}^{\alpha, p}\left(\mathbb{R}^{n}\right)} \leq C N^{n / r}\left\|\phi_{+}^{*}(f)\right\|_{\dot{K}_{q(\cdot)}^{\alpha, p}\left(\mathbb{R}^{n}\right)}
$$

for any $r \in(0,1)$.

To prove (3.1) we first show that if $x \in E_{\varepsilon}$, then

$$
u_{\varepsilon, l, N}^{*}(x) \leq C N^{n / r}\left(M\left[\phi_{+}^{*}(f)\right]^{r}(x)\right)^{1 / r} .
$$

Note that for each fixed $x \in E_{\varepsilon}$, there exists $(y, t) \in \mathbb{R}_{+}^{n+1}$ such that $|x-y|<$ $N t<1 / \varepsilon$ and

$$
\left|\left(f * \phi_{t}\right)(x)\right| \geq\left|\left(f * \phi_{t}\right)(y)\right|\left(\frac{N t}{N t+\varepsilon}\right)^{l}(1+\varepsilon N|y|)^{-l}>u_{\varepsilon, l, N}^{*}(x) / 2 .
$$

On the other hand, we know by the definition of $E_{\varepsilon}$ that if $x \in E_{\varepsilon}$ and $|x-z|<N t$, then

$$
t\left|\nabla_{z}\left(f * \phi_{t}\right)(z)\right| \leq C_{0}\left(\frac{N t}{N t+\varepsilon}\right)^{-l}(1+\varepsilon l|y|)^{l} u_{\varepsilon, l, N}^{*}(x) .
$$

Therefore, if $x \in E_{\varepsilon},|x-y|<N t$ and $|x-z|<N t$, then $t\left|\nabla_{z}\left(f * \phi_{t}\right)(z)\right| \leq$ $C_{1}\left|\left(f * \phi_{t}\right)(y)\right|$. Applying the mean value theorem, we have that for $w \in B(x, N t) \cap$ $B\left(y, t /\left(2 C_{1}\right)\right)$,

$$
\left|\left(f * \phi_{t}\right)(w)-\left(f * \phi_{t}\right)(y)\right| \leq\left|\nabla_{z}\left(f * \phi_{t}\right)(z)\right||w-y| \leq\left|\left(f * \phi_{t}\right)(y)\right| / 2,
$$

where $z=\theta w+(1-\theta) y$ and $\theta \in(0,1)$. This shows that if $x \in E_{\varepsilon}$ and $w \in$ $B(x, N t) \cap B\left(y, t /\left(2 C_{1}\right)\right)$, then

$$
\left|\left(f * \phi_{t}\right)(w)\right| \geq\left|\left(f * \phi_{t}\right)(y)\right| / 2 \geq u_{\varepsilon, l, N}^{*}(x) / 4 .
$$


Thus, for any $r \in(0,1)$ and $x \in E_{\varepsilon}$, we have

$$
\begin{aligned}
M\left(\left(\phi_{+}^{*}(f)\right)^{r}\right)(x) & \geq \frac{1}{|B(x, N t)|} \int_{B(x, N t)}\left(\phi_{+}^{*}(f)(w)\right)^{r} d w \\
& \geq \frac{1}{|B(x, N t)|} \int_{B(x, N t) \cap B\left(y, t /\left(2 C_{1}\right)\right)}\left|f * \phi_{t}(w)\right|^{r} d w \\
& \geq C N^{-n}\left(u_{\varepsilon, l, N}^{*}(x)\right)^{r},
\end{aligned}
$$

and so (3.2) is true. Now choosing $r$ sufficiently small so that $0<r \alpha<n \delta_{2}$, then by (3.2) and Lemma 1.6 we have

$$
\begin{aligned}
\left\|u_{\varepsilon, l, N}^{*} \chi_{E_{\varepsilon}}\right\|_{\dot{K}_{q(\cdot)}^{\alpha, p}\left(\mathbb{R}^{n}\right)} \leq C N^{n / r}\left\|\left(M\left(\phi_{+}^{*}(f)\right)^{r}\right)^{1 / r}\right\|_{\dot{K}_{q(\cdot)}^{\alpha, p}\left(\mathbb{R}^{n}\right)} & =C N^{n / r}\left\|M\left(\phi_{+}^{*}(f)\right)^{r}\right\|_{\dot{K}_{q(\cdot) / r}^{\alpha r, p / r}\left(\mathbb{R}^{n}\right)}^{1 / r} \\
& \leq C N^{n / r}\left\|\phi_{+}^{*}(f)\right\|_{\dot{K}_{q(\cdot)}^{\alpha, p}\left(\mathbb{R}^{n}\right) \cdot}
\end{aligned}
$$

This completes the proof of (iv) $\Rightarrow($ ii). Moreover,

$$
\left\|\phi_{\nabla, N}^{*}(f)\right\|_{\dot{K}_{q(\cdot)}^{\alpha, p}\left(\mathbb{R}^{n}\right)} \leq C N^{n / r}\left\|\phi_{+}^{*}(f)\right\|_{\dot{K}_{q(\cdot)}^{\alpha, p}\left(\mathbb{R}^{n}\right)} .
$$

Now we consider (iv) $\Rightarrow(\mathrm{i})$. By a simple computation, we know that

$$
\phi_{M}^{* *}(x) \leq \phi_{\nabla}^{*}(f)(x)+\sum_{k=0}^{\infty} 2^{-k M} \phi_{\nabla, 2^{k+1}}^{*}(f)(x) .
$$

This via Lemma 3.2 and (3.3) gives that if $N$ is large enough, then

$$
\begin{aligned}
\left\|G_{N}(f)\right\|_{\dot{K}_{q(\cdot)}^{\alpha, p}\left(\mathbb{R}^{n}\right)} & \leq C\left\|\phi_{\nabla}^{*}(f)\right\|_{\dot{K}_{q(\cdot)}^{\alpha, p}\left(\mathbb{R}^{n}\right)}+C \sum_{k=0}^{\infty} 2^{-k M}\left\|\phi_{\nabla, 2^{k+1}}^{*}(f)\right\|_{\dot{K}_{q(\cdot)}^{\alpha, p}\left(\mathbb{R}^{n}\right)} \\
& \leq C \sum_{k=0}^{\infty} 2^{-k(M-n / r)}\left\|\phi_{+}^{*}(f)\right\|_{\dot{K}_{q(\cdot)}^{\alpha, p}\left(\mathbb{R}^{n}\right)} \\
& \leq C\left\|\phi_{+}^{*}(f)\right\|_{\dot{K}_{q(\cdot)}^{\alpha, p}\left(\mathbb{R}^{n}\right)},
\end{aligned}
$$

where $M>n / r$. Thus (iv) $\Rightarrow$ (i) holds and the proof of Theorem 3.3 is completed.

Remark 3.4. From the proof of Theorem 3.3 we can see that for any $N_{1}, N_{2}>$ $n+1$, the set

coincide with the set

$$
\left\{f \in \mathcal{S}\left(\mathbb{R}^{n}\right): G_{N_{1}}(f) \in \dot{K}_{q(\cdot)}^{\alpha, p}\left(\mathbb{R}^{n}\right)\right\}
$$

$$
\left\{f \in \mathcal{S}\left(\mathbb{R}^{n}\right): G_{N_{2}}(f) \in \dot{K}_{q(\cdot)}^{\alpha, p}\left(\mathbb{R}^{n}\right)\right\} .
$$

Moreover

$$
\left\|G_{N_{1}}(f)\right\|_{\dot{K}_{q(\cdot)}^{\alpha, p}\left(\mathbb{R}^{n}\right)} \approx\left\|G_{N_{2}}(f)\right\|_{\dot{K}_{q(\cdot)}^{\alpha, p}\left(\mathbb{R}^{n}\right)} .
$$

The same conclusions are also true for non-homogeneous space.

Now we will give another characterization of spaces $H \dot{K}_{q(\cdot)}^{\alpha, p}\left(\mathbb{R}^{n}\right)$ and $H K_{q(\cdot)}^{\alpha, p}\left(\mathbb{R}^{n}\right)$. Given $s \in(0,1) \cup \mathbb{Z}_{+}$, define $\mathcal{T}_{s}$ to be the space of $C^{\infty}$ functions on $\mathbb{R}^{n}$ with support contained in $B(0,1)$ such that $|\varphi(x)-\varphi(y)| \leq|x-y|^{s}$, for all $x, y \in \mathbb{R}^{n}$, 
when $s \in(0,1)$ and $\sum_{j=1}^{s}\left\|\nabla^{j} \varphi\right\|_{\infty} \leq 1$, when $s \in \mathbb{Z}_{+}$. Let $f_{s}^{*}=\sup _{t>0} \sup _{\varphi \in \mathcal{T}_{s}}\left|\varphi_{t} * f(x)\right|$, for $s \in(0,1) \cup \mathbb{Z}_{+}$, and set $\mathcal{T}_{1}=\mathcal{T}$ and $f_{1}^{*}(x)=f^{*}(x)$.

Theorem 3.5. Let $n \delta_{2} \leq \alpha<\infty, 0<p<\infty$ and $q(\cdot) \in \mathcal{B}\left(\mathbb{R}^{n}\right)$. Suppose $s \in(0,1) \cup \mathbb{Z}_{+}$and $s>\alpha / \delta_{2}-n$. Then $f \in H \dot{K}_{q(\cdot)}^{\alpha, p}\left(\mathbb{R}^{n}\right)$ (or $\left.H K_{q(\cdot)}^{\alpha, p}\left(\mathbb{R}^{n}\right)\right)$ if and only if $f_{s}^{*} \in \dot{K}_{q(\cdot)}^{\alpha, p}\left(\mathbb{R}^{n}\right)$ (or $\left.K_{q(\cdot)}^{\alpha, p}\left(\mathbb{R}^{n}\right)\right)$.

The proof of Theorem 3.5 is based on Theorem 3.3 and the following two lemmas.

Lemma 3.6. Let $n \delta_{2} \leq \alpha<\infty, 0<p<\infty$ and $q(\cdot) \in \mathcal{B}\left(\mathbb{R}^{n}\right)$. If $\sigma, s \in(0,1) \cup \mathbb{Z}_{+}$ and $\alpha / \delta_{2}-n<\sigma<s$, then there are constants $C_{2}, C_{3}>0$ such that

$$
C_{2}^{-1}\left\|f_{\sigma}^{*}\right\|_{\dot{K}_{q(\cdot)}^{\alpha, p}\left(\mathbb{R}^{n}\right)} \leq\left\|f_{s}^{*}\right\|_{\dot{K}_{q(\cdot)}^{\alpha, p}\left(\mathbb{R}^{n}\right)} \leq C_{2}\left\|f_{\sigma}^{*}\right\|_{\dot{K}_{q(\cdot)}^{\alpha, p}\left(\mathbb{R}^{n}\right)}
$$

and

$$
C_{3}^{-1}\left\|f_{\sigma}^{*}\right\|_{K_{q(\cdot)}^{\alpha, p}\left(\mathbb{R}^{n}\right)} \leq\left\|f_{s}^{*}\right\|_{K_{q(\cdot)}^{\alpha, p}\left(\mathbb{R}^{n}\right)} \leq C_{3}\left\|f_{\sigma}^{*}\right\|_{K_{q(\cdot)}^{\alpha, p}\left(\mathbb{R}^{n}\right)}
$$

for all distributions $f$ on $\mathbb{R}^{n}$.

Proof. We only prove the homogeneous case. Note that $\sigma>\alpha / \delta_{2}-n$. We can choose $q_{1}$ to satisfy $\frac{n}{\sigma+n}<q_{1}<\frac{n \delta_{2}}{\alpha}$. Since $n \delta_{2} \leq \alpha<\infty$, we have $n \delta_{2} / \alpha \leq 1$. So $0<q_{1}<1$. Setting $\varphi \in \mathcal{T}_{\sigma}$, from [5] we know $f_{\sigma}^{*} \leq C M\left(\left(f_{s}^{*}\right)^{q_{1}}\right)^{1 / q_{1}}$. Therefore, by Lemma 1.6 we have

$$
\begin{aligned}
\left\|f_{\sigma}^{*}\right\|_{\dot{K}_{q(\cdot)}^{\alpha, p}\left(\mathbb{R}^{n}\right)} & =\left\{\sum_{k=-\infty}^{\infty} 2^{k \alpha p}\left\|f_{\sigma}^{*} \chi_{k}\right\|_{L^{q(\cdot)}\left(\mathbb{R}^{n}\right)}^{p}\right\}^{1 / p} \\
& \leq C\left\{\sum_{k=-\infty}^{\infty} 2^{k \alpha p}\left\|M\left(\left(f_{s}^{*}\right)^{q_{1}}\right) \chi_{k}\right\|_{L^{q(\cdot) / q_{1}\left(\mathbb{R}^{n}\right)}}^{p / q_{1}}\right\}^{1 / p} \\
& =C\left\|M\left(\left(f_{s}^{*}\right)^{q_{1}}\right) \chi_{k}\right\|_{\dot{K}_{q(\cdot) / q_{1}}^{\alpha q_{1}, p / q_{1}}\left(\mathbb{R}^{n}\right)}^{q_{1}} \leq C\left\|f_{s}^{*}\right\|_{\dot{K}_{q(\cdot)}^{\alpha, p} .}
\end{aligned}
$$

On the other hand, it follows from the definition of $f_{s}^{*}$ that $f_{s}^{*}(x) \leq f_{\sigma}^{*}(x)$. From this, we deduce the conclusion of Lemma 3.6.

Remark 3.7. Let $p, q(\cdot)$ be as in Lemma 3.6. If $\alpha=n \delta_{2}, \sigma \in(0,1)$ and $s \in \mathbb{Z}_{+}$, then there are constants $C_{4}, C_{5}>0$ such that

$$
C_{4}^{-1}\left\|f_{\sigma}^{*}\right\|_{\dot{K}_{q(\cdot)}^{\alpha, p}\left(\mathbb{R}^{n}\right)} \leq\left\|f_{s}^{*}\right\|_{\dot{K}_{q(\cdot)}^{\alpha, p}\left(\mathbb{R}^{n}\right)} \leq C_{4}\left\|f^{*}\right\|_{\dot{K}_{q(\cdot)}^{\alpha, p}\left(\mathbb{R}^{n}\right)} \leq C_{4}\left\|f_{\sigma}^{*}\right\|_{\dot{K}_{q(\cdot)}^{\alpha, p}\left(\mathbb{R}^{n}\right)}
$$

and

$$
C_{5}^{-1}\left\|f_{\sigma}^{*}\right\|_{K_{q(\cdot)}^{\alpha, p}\left(\mathbb{R}^{n}\right)} \leq\left\|f_{s}^{*}\right\|_{K_{q(\cdot)}^{\alpha, p}\left(\mathbb{R}^{n}\right)} \leq C_{5}\left\|f^{*}\right\|_{K_{q(\cdot)}^{\alpha, p}\left(\mathbb{R}^{n}\right)} \leq C_{5}\left\|f_{\sigma}^{*}\right\|_{K_{q(\cdot)}^{\alpha, p}\left(\mathbb{R}^{n}\right)}
$$

for all distributions $f$ on $\mathbb{R}^{n}$.

Let $\theta(z)$ be a bump function which satisfies $\theta \in C_{0}^{\infty}\left(\mathbb{R}^{n}\right), \operatorname{supp} \theta \subset B(0,1)$, and $\int_{\mathbb{R}^{n}} \theta(x) d x=1$. 
Lemma 3.8. Let $n \delta_{2} \leq \alpha<\infty, 0<p<\infty$ and $q(\cdot) \in \mathcal{B}\left(\mathbb{R}^{n}\right)$. Suppose $f \in \mathcal{S}^{\prime}\left(\mathbb{R}^{n}\right), \theta$ is above and $\theta_{+}^{*}(f)$ is as in Theorem 3.3. If $s \in \mathbb{Z}_{+}, \sigma \in(0,1) \cup \mathbb{Z}_{+}$ and $\alpha / \delta_{2}-n<\sigma$, then there are constants $C_{6}, C_{7}>0$ such that

$$
C_{6}^{-1}\left\|f_{\sigma}^{*}\right\|_{\dot{K}_{q(\cdot)}^{\alpha, p}\left(\mathbb{R}^{n}\right)} \leq\left\|\theta_{+}^{*}(f)\right\|_{\dot{K}_{q(\cdot)}^{\alpha, p}\left(\mathbb{R}^{n}\right)} \leq C_{6}\left\|f_{s}^{*}\right\|_{\dot{K}_{q(\cdot)}^{\alpha, p}\left(\mathbb{R}^{n}\right)}
$$

and

$$
C_{7}^{-1}\left\|f_{\sigma}^{*}\right\|_{K_{q(\cdot)}^{\alpha, p}\left(\mathbb{R}^{n}\right)} \leq\left\|\theta_{+}^{*}(f)\right\|_{K_{q(\cdot)}^{\alpha, p}\left(\mathbb{R}^{n}\right)} \leq C_{7}\left\|f_{s}^{*}\right\|_{K_{q(\cdot)}^{\alpha, p}\left(\mathbb{R}^{n}\right)} .
$$

Proof. The method of proof is similar to [5, Lemma 2.2]. Here we omit it.

Remark 3.9. Let $p, q(\cdot), \theta$ and $\theta_{+}^{*}(f)$ be as in Lemma 3.3. If $\alpha=n \delta_{2}, \sigma \in(0,1)$ and $s \in \mathbb{Z}_{+}$, then there are constants $C_{8}, C_{9}>0$ such that

$$
C_{8}^{-1}\left\|f_{\sigma}^{*}\right\|_{\dot{K}_{q(\cdot)}^{\alpha, p}\left(\mathbb{R}^{n}\right)} \leq\left\|\theta_{+}^{*}(f)\right\|_{\dot{K}_{q(\cdot)}^{\alpha, p}\left(\mathbb{R}^{n}\right)} \leq C_{8}\left\|f_{s}^{*}\right\|_{\dot{K}_{q(\cdot)}^{\alpha, p}\left(\mathbb{R}^{n}\right)}
$$

and

$$
C_{9}^{-1}\left\|f_{\sigma}^{*}\right\|_{K_{q(\cdot)}^{\alpha, p}\left(\mathbb{R}^{n}\right)} \leq\left\|\theta_{+}^{*}(f)\right\|_{K_{q(\cdot)}^{\alpha, p}\left(\mathbb{R}^{n}\right)} \leq C_{9}\left\|f_{s}^{*}\right\|_{K_{q(\cdot)}^{\alpha, p}\left(\mathbb{R}^{n}\right)} .
$$

Acknowledgement. The authors are very grateful to the referees for their valuable comments. This work was supported by the National Natural Science Foundation of China (Grant Nos. 11171345 and 11101423).

\section{REFERENCES}

1. C. Capone, D. Cruz-Uribe and A. Fiorenza, The fractional maximal operator and fractional integrals on variable $L^{p}$ spaces, Rev. Mat. Iberoamericana 23 (2007), 743-770.

2. C. Fefferman and E.M. Stein, $H^{p}$ spaces of several variables, Acta. Math. 129 (1973), $137-193$.

3. M. Izuki, Boundedness of sublinear operators on Herz spaces with variable exponent and application to wavelet characterization, Anal. Math. 36 (2010), 33-50.

4. O. Kováčik and J. Rákosník, On spaces $L^{p(x)}$ and $W^{k, p(x)}$, Czechoslovak Math. J. 116 (1991), 592-618.

5. S. Lu and D. Yang, Some characterizations of weighted Herz-type Hardy spaces and their applications, Acta Math. Sin. (Engl. Ser.) 13 (1997), 45-58.

6. H. Wang, The decomposition for the Herz space with variable exponent and its applications, 1425-1431, Proceedings of Academic Conference on Research Achievements of the Fundamental Research Funds for the Central Universities, China Coal Industry Publishing House, 2011.

7. H. Wang and Z. Liu, The Herz-type Hardy spaces with variable exponent and its applications, Taiwanese J. Math. 16 (2012), 1363-1389.

1 Department of Mathematical and Physical Science, Zibo Normal College, Zibo 255130, Shandong, P. R. China.

E-mail address: hbwang_2006@163.com

2 Department of Mathematics, China University of Mining and Technology(Beijing), BeiJing 100083, P. R. China.

E-mail address: liuzg@cumtb.edu.cn 